\title{
NLRP1 and NLRP3 inflammasomes mediate LPS/ATP-induced pyroptosis in knee osteoarthritis
}

\author{
LING-RUI ZHAO, RUN-LIN XING, PEI-MIN WANG, NONG-SHAN ZHANG, \\ SONG-JIANG YIN, XIAO-CHEN LI and LI ZHANG
}

\begin{abstract}
Department of Orthopaedics, Affiliated Hospital of Nanjing University of Traditional Chinese Medicine, Nanjing, Jiangsu 210029, P.R. China
\end{abstract}

Received October 7, 2017; Accepted January 23, 2018

DOI: $10.3892 / \mathrm{mmr} .2018 .8520$

\begin{abstract}
Pyroptosis is triggered by inflammasomes after its activation by various inflammatory stimulations, including lipopolysaccharide (LPS) and improper $\mathrm{pH}$. This may result in programmed death of the affected cell. It is well known that NLRP1 and NLRP3 inflammasomes mediate the production of various cytokines in inflammatory disorders; however, it is still unknown whether NLRP1 and NLRP3 inflammasomes can influence the LPS-induced pyroptosis in the progression of knee osteoarthritis (KOA). In the present study, the correlation between the NLRP inflammasomes and fibroblast-like synoviocytes (FLSs) pyroptosis was investigated in vivo and in vitro. Human synovial samples were collected from KOA patients and the expression of NLRP1 and NLRP3 inflammasomes was analyzed. Human FLS were isolated in vitro and stimulated with LPS. To determine whether NLRP1 and NLRP3 inflammasomes are involved in FLS pyroptosis, NLRP1 and NLRP3 small interfering RNAs (siRNAs) were used. The results showed that the expression of NLRPs and inflammasome-related proteins were upregulated and FLS stimulated with LPS+ATP resulted in cell pyroptosis. However, LPS+ATP-induced pyroptosis was attenuated by NLRP1 and NLRP3 siRNAs. The results of the present study indicate that LPS-induced FLS pyroptosis may be mediated by either NLRP1 or NLRP3 inflammsomes. Overall, based on the data obtained from patients and in vitro
\end{abstract}

Correspondence to: Dr Pei-Min Wang, Department of Orthopaedics, Affiliated Hospital of Nanjing University of Traditional Chinese Medicine, 155 Hanzhong Road, Nanjing, Jiangsu 210029, P.R. China E-mail:drwpm@163.com

Abbreviations: KOA, knee osteoarthritis; FLS, fibroblast-like synoviocytes; NLRP, nod-like receptor protein; LPS, lipopolysaccharide; ATP, adenosine triphosphate; ASC, apoptosis-associated speck-like protein with a caspase-recruitment domain; GSDMD, gasdermin D; siRNAs, small interfering RNAs; RT-qPCR, reverse transcription-quantitative polymerase chain reaction; SDS-PAGE, sodium dodecyl sulphate polyacrylamide gel electrophoresis

Key words: inflammasome, pyroptosis, knee osteoarthritis, synovium, fibroblast-like synoviocytes cells, the present finsings showed that NLRP1 and NLRP3 inflammasomes are highly involved in the FLS inflammation and pyroptosis. Furthermore, inhibition of NLRP1 and NLRP3 led to a remarkable reduction of pyroptosis-related cytokines. Thus, NLRP1 and NLRP3 inflammasomes may be important in the pathogenesis of OA and may represent a novel therapeutic target.

\section{Introduction}

Knee osteoarthritis (KOA) is a chronic and degenerative disease that threatens global health. With increased population aging, the incidence of KOA is growing at an alarming rate (1). Occurring predominantly in the elderly, it is manifested by pain and articular function disorder (2) in clinic, cartilage degeneration, subchondral sclerosis and osteophyte formation in pathology. Among the recent research outcomes, novel biological markers have become more promising tools or indicators to assess the severity and progress of local KOA (3).

The fibroblast-like synoviocyte (FLS) has been identified to involve in the progression of KOA, it may play a crucial role via the secretion of various of pro-inflammatory cytokines, such as IL-1 $\beta$, IL-18 and TNF- $\alpha$. These cytokines may directly participate and induce the local synovial inflammation and cartilage matrix degradation. IL- $1 \beta$ can activate the expression of Transient Receptor Proteins (TRPs), which may lead to both thermal and mechanical hyperalgesia (4-5). IL-18 has been identified as the trigger of cartilage proteoglycan degradation. TNF- $\alpha$ may regulate the sensitization of thermal and mechanical nociceptors through activating the related TRP channels (6). Current studies toward these cytokines mostly focus on the inflammation-related cell signal pathways, yet the clinical treatments still remain unsatisfactory. Further hypothesis and research are needed to achieve the clinical goal (7-10).

Cell apoptosis was classically defined as the autonomous and programmed death controlled by genes in order to maintain a stable internal environment. Chondrocyte and FLS apoptosis is an active process involving the activation, expression and regulation of many genes (11), they may be the main cause of synovium inflammation or clinical symptoms.

Inflammasomes are intracellular multi-protein complexes that can be activated by many exogenous and endogenous factors, such as lipopolysaccharide (LPS), oxidative stress, 
potassium efflux, and monosodium urate crystals (12). Inflammasome activation triggers the maturation and expression of proinflammatory cytokines, such as IL-1 $\beta$ and IL-18 mentioned above, to initiate innate immune responses and subsequently induce cellular injury or even death (13). The inflammasome-induced cell death has been considered as a non-canonical programmed cell death which mainly occurs in the inflammatory conditions (14). It is also defined as 'cell pyroptosis'. NLRP1 and NLRP3 inflammasomes are the most thoroughly researched among all types of inflammasomes.

The NLRP inflammasomes consist of NLRP1/3 receptors, apoptosis-associated Speck-like protein with a caspase-recruitment domain (ASC) and pro-caspase-1 (caspase-1-induced pyroptosis is an innate immune effector mechanism against intracellular bacteria). The NLRP inflammasome is formed after the oligomerization of NLRP receptors and subsequent recruitment of adaptor ASC and pro-caspase-1. The activation of NLRP receptors inflammasome finally triggers the maturation of IL-1 $\beta$ and IL-18 via activated caspase-1 $(15,16)$. Meanwhile, various of inflammatory cytokines may also rapidly overflow, such as high mobility group box proteins (HMGBs). Finally, the activation of NF- $\mathrm{B}$ and other canonical inflammatory signaling pathways may result in a cascaded release of inflammatory factors, further promote the rapid progress of inflammatory response $(17,18)$.

$\mathrm{KOA}$ is a common aseptic inflammation disease, cell apoptosis may participated in the entire pathogenesis of KOA. However, the programmed cell death should be regulated by certain signaling pathways without inducing any inflammatory reactions. Cell pyroptosis is another form of programmed inflammatory cell death, when cell apoptosis cannot be activated properly and cell death indeed occurs. In the present study, we investigated the correlation between the NLRP inflammasomes, OA inflammation and FLS pyroptosis in patients' tissue and in vitro cells via molecular biology and histochemical methods (19). Our hypothesis is that NLRP1 and NLRP3 inflammasomes are crucial mediators of FLS pyroptosis in KOA. Inhibition of NLRP inflammasome may be exert a protective effect.

\section{Patients and methods}

Patients and synovial samples. Human synovial samples were collected with informed consent in line with the Declaration of Helsinki (2000 revision). The study protocol was approved by the local Ethics Committee of Affiliated Hospital of Nanjing University of Traditional Chinese Medicine (Jiangsu, China; approvalno.2015NL-068-02). The diagnosis of OA was based on clinicalandradiologicalevidenceofdegenerativechangesduring surgery. Synovial tissues were obtained under aseptic conditions from $10 \mathrm{OA}$ patients undergoing total knee replacement surgery. Samples of non-arthritic synovial tissues wereobtainedatarthroscopy followingtrauma/jointderangement.

All the patients in this study were enrolled from the Department of Orthopedic Surgery, The First Affiliated Hospital of Nanjing University of Chinese Medicine (Jiangsu, China). Data from a medical history and physical examination were compiled for each patient. A total of $50 \%$ of the enrolled patients were receiving non-steroidal anti-inflammatory drugs (NSAIDs) and 50\% were not on medication.
Culture and treatment of FLSs. Synovial tissues were minced into pieces of $2 \sim 3 \mathrm{~mm}^{2}$, homogenized in DMEM and then incubated overnight at $37^{\circ} \mathrm{C}$ with $1 \mathrm{mg} / \mathrm{ml}$ type I collagenase and $1 \mathrm{mg} / \mathrm{ml}$ type II dispase (both Sigma-Aldrich, St. Louis, MO, USA). Following cell dissociation, the samples were filtered through a cell strainer. After dissociation, fibroblasts were pelleted by centrifugation at $1,000 \mathrm{rpm}$ for $8 \mathrm{~min}$ and plated in DMEM supplemented with 10\% FCS (Gibco; Thermo Fisher Scientific, Waltham, MA, USA) and antibiotics (100 U/ml penicillin, $100 \mathrm{ug} / \mathrm{ml}$ streptomycin; Invitrogen, Carlsbad, CA, USA). Cells were cultured at $37^{\circ} \mathrm{C}$ in a humidified $95 \%$ air and $5 \% \mathrm{CO}_{2}$ atmosphere. The cell confluence and morphology were assessed throughout the experiments by immunohistochemical analysis. All the functional experiments were conducted using primary synovial cultured cells from passages 3-6.

FCL were stimulated with LPS (1 ug/ml) in DMEM for $6 \mathrm{~h}$ to stimulate the inflammatory reactions, and they were then challenged with ATP ( $3 \mathrm{mM})$ for $1 \mathrm{~h}$ to activate the NLRP1 and NLRP3 inflammasome. The FCL exposed to DMEM with same volume of saline served as controls $(20,21)$.

Small-interfering RNA preparation and transfection. To inhibit the NLRP1 and NLRP3 expression in the FLS, commercially available NLRP1, NLRP3 and vehicle control small interfering RNA (siRNA; Invitrogen) were used. FLSs were transfected with siRNAs by using Lipofectamine 2000 (Invitrogen) according to the manufacturer's instructions. siRNA was diluted in transfection reagent and culture medium, and the cells were incubated with 20 pmol siRNA for $6 \mathrm{~h}$.

Reverse transcription-quantitative polymerase chain reaction (RT-qPCR). Total RNA was extracted from synovial tissues and FLSs by using TRIzol (Invitrogen). The reverse transcription was performed by using a first strand cDNA synthesis kit (Takara, Otsu, Japan) according to the manufacturer's instructions. qPCR was performed using Premix Ex Taq SYBR-Green PCR (Takara) according to the manufacturer's instructions on an ABI PRISM 7300 (Applied Biosystems, Foster City, CA, USA). The sequences of the primers were as follows: caspase-1 forward, 5'-CTACTTCCCTGAATGCTTGGC-3' and reverse, 5'-GCTCCTGGGTTTGTCCACTC-3'; NLRP1 forward, 5'-GCATGGGGACCTGACATGGG-3' and reverse, 5'-GATGCAATCTCAGCACCGGAA-3'; NLRP3 forward, 5'-GCTCATATCATCATTCCCGCT-3' and reverse, 5'-ACC AGCTACAAAAAGCATGGA-3'; ASC forward, 5'-CCAAAT GCTTCCCCCATCCT-3' and reverse, 5'-GCCCTTTGGTAC ATGCCTCT-3'; GSDMD forward, 5'-TCACAACCTTGG GGCATCAG-3' and reverse, 5'-TCCTTCCTGCAAGCTGGT TC-3'; IL-18 forward, 5'-TGCTGCAGTCTACACAGCTT-3' and reverse, 5'-TTGTTGCGAGAGGAAGCGAT-3'GADPH; forward, 5'-ACGGGAAGCTTGTCATCAAT-3' and reverse, 5'-TGGAAGATGGTGATGGGATT-3. GAPDH was used as internal control.

Western blot analysis. Synovial tissues were dissected, weighed and mixed with RIPA lysate. Samples were centrifuged at $4^{\circ} \mathrm{C}$ with $15,000 \mathrm{r} / \mathrm{min}$ for $15 \mathrm{~min}$. The supernatant was removed. Cultured cells were washed and lysed. Then the protein levels were quantified with a BCA protein Assay kit (Roche, Basel, Switzerland). The protein samples were electrophoresed in 
Table I. Information and histological diagnoses of selected patients.

\begin{tabular}{clccc}
\hline Number & Sex & $\begin{array}{c}\text { Age } \\
\text { (years) }\end{array}$ & $\begin{array}{c}\text { KOA duration } \\
\text { (years) }\end{array}$ & $\begin{array}{c}\text { Receiving } \\
\text { NSAIDs }\end{array}$ \\
\hline 1 & Female & 51 & 6 & Yes \\
2 & Female & 75 & 15 & Yes \\
3 & Female & 52 & 2 & No \\
4 & Male & 65 & 7 & No \\
5 & Male & 54 & 10 & Yes \\
6 & Female & 41 & 5 & No \\
7 & Female & 59 & 7 & Yes \\
8 & Male & 63 & 8 & No \\
9 & Male & 77 & 20 & Yes \\
10 & Female & 65 & 2 & No \\
\hline
\end{tabular}

KOA, knee osteoarthritis; NSAIDS, non-steroidal anti-inflammatory drugs.

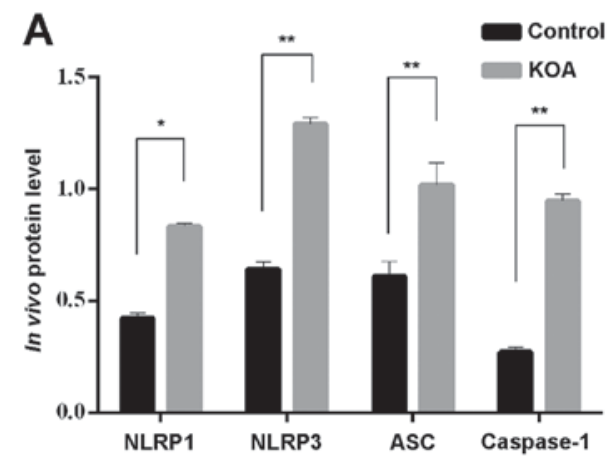

B

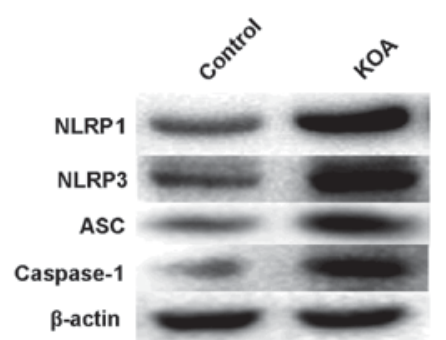

Figure 1. (A) Increased protein expression levels of NLRP1 and NLRP3 inflammasomes in synovia of KOA patients. The total proteins were extracted from normal and KOA synovia the results are expressed as the mean $\pm \mathrm{SD}$. ${ }^{*} \mathrm{P}<0.05$ compared with KOA synovia. ${ }^{* *} \mathrm{P}<0.01$ compared with KOA synovia. (B) Protein expression of NLRP1, NLRP3, ASC and caspase-1 in KOA synovia were upregulated comparing with control group. KOA, knee osteoarthritis; ASC, apoptosis-associated Speck-like protein with a caspase-recruitment domain.

SDS-PAGE to separate protein bands. Proteins were transferred from gel onto PVDF membrane, blocked with 5\% non-fat dry milk for $2 \mathrm{~h}$. The membrane was incubated with monoclonal rabbit antibodies specific for human NLRP1, NLRP3, ASC, caspase-1, IL-18, GSDMD, $\beta$-actin and GAPDH $(1: 1,000$; Invitrogen), overnight at $4^{\circ} \mathrm{C}$. On the next day, membranes were incubated with second antibody (1:5,000; Invitrogen) for $2 \mathrm{~h}$. The bands were visualized by ECL method (Invitrogen) and the overall gray value of protein bands (average gray value $\mathrm{x}$ gray value area) was quantified with Photoshop CS5 (Adobe Systems, Inc., San Jose, CA, USA) software to calculate the target protein relative value (target protein gray value/internal reference overall gray value).

Terminal deoxynucleotidyl transferase dUTP nick end labeling (TUNEL assay). Apoptosis in tissue was assessed using a TUNEL assay and identified using the in situ cell death detection kit (Roche) according to the protocol. In brief, sections were deparaffinized using xylene reagent. The deparaffinized sections were then incubated with proteinase $\mathrm{K}$ $(30 \mathrm{~g} / \mathrm{ml})$, followed by treatment with $0.3 \%$ hydrogen peroxide for $30 \mathrm{~min}$ at room temperature. Incubation with the TUNEL reaction mixture was performed in a humidified chamber at $37^{\circ} \mathrm{C}$. Converter-POD was added for signal conversion and incubated in a 3,3-diaminobenzidine reaction mixture, which served as the chromogen. After dehydration, the sections were mounted in DPX.

$H \& E$ and immunostaining. Both H\&E staining and immunostaining were applied to observe the morphology of FLS. The cells were washed with PBS and treated with blocking buffer containing $1 \%$ BSA and $0.2 \%$ Triton $\mathrm{X}$ in PBS for $1 \mathrm{~h}$ at room temperature. The samples were then incubated overnight at $4^{\circ} \mathrm{C}$ with anti-vimentin rabbit polyclonal antibody (Invitrogen). After washing with PBS, the secondary antibody was applied, and the signals were visualized using an ABC kit (Santa Cruz Biotechnology, Dallas, TX, USA).

Flow cytometry. After treatment, the cells were double stained with Annexin V-fluorescein isothiocyanate and propidium iodide (Annexin V:FITC apoptosis detection kit; BD Biosciences, Santa Cruz, CA, USA) according to the instructions. Quantification was then performed by flow cytometry (Beckman Coulter, Miami, FL, USA).

Statistical analysis. Statistical analysis was performed using GraphPad Prism 6.0 Software (San Diego, CA, USA). All data shown in bar graphs are mean $\pm \mathrm{SD}$. Data were analyzed by one-way or two-way ANOVA with Bonferroni's or Dunnett's post hoc test for comparison of multiple columns (as appropriate).

\section{Results}

Expression of NLRPI and NLRP3 inflammasomes are upregulated in the synovia of KOA patients. As inflammation is an important part of KOA progression, we examined the protein and mRNA levels of NLRP1 and NLRP3 in the synovia of KOA patients. Patients with KOA were included in this study, as shown in Table I. The protein and mRNA expression of both NLRP1 and NLRP3 inflammasomes (consist of NLRP1/NLRP3+ASC+caspase-1) were significantly upregulated in the KOA patient tissues compared with controls (Figs. 1 and 2), TUNEL staining revealed the TUNEL-positive cells in the KOA region (Fig. 3).

$L P S+A T P$ induced cell programmed death leads to the activation of NLRP1 and NLRP3 inflammasomes. The FLSs 


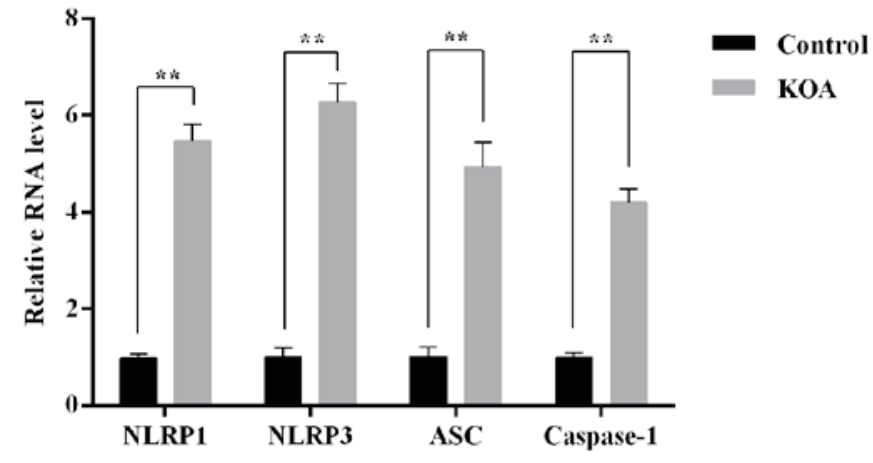

Figure 2. Increased protein expression levels of NLRP1 and NLRP3 inflammasomes in synovia of KOA patients. The total mRNAs were extracted from normal and KOA synovia. The results are expressed as the mean $\pm \mathrm{SD}$. ${ }^{* *} \mathrm{P}<0.01$ compared with KOA synovia. KOA, knee osteoarthritis.
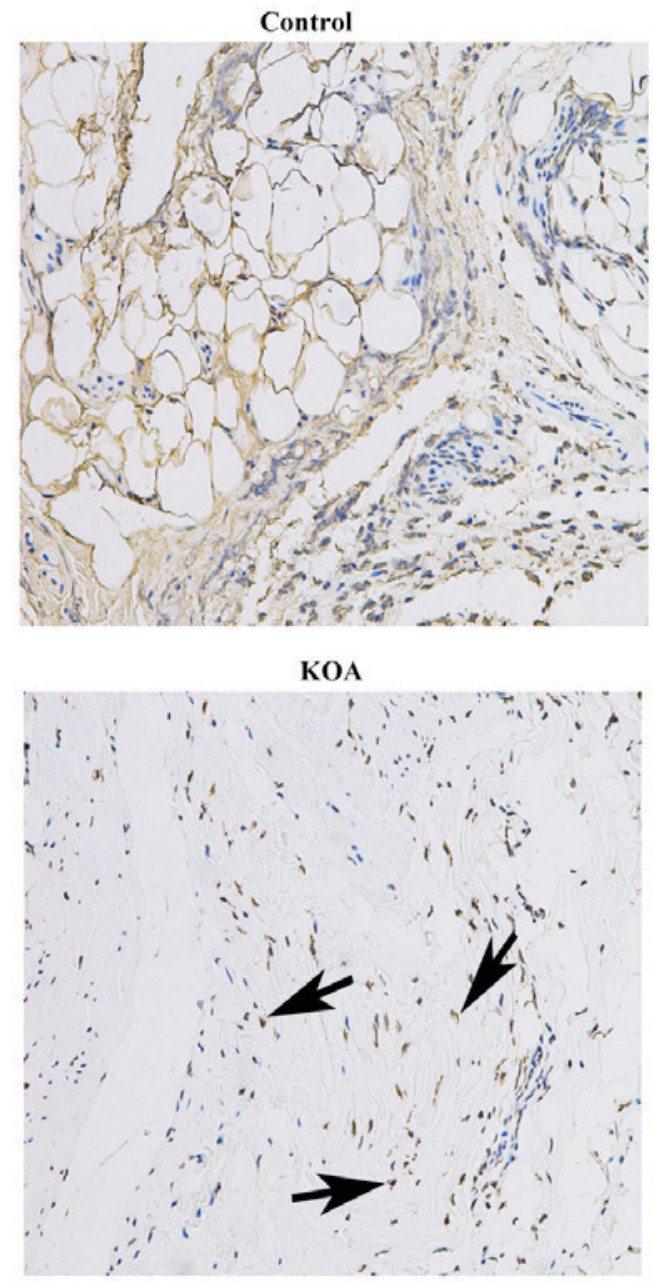

Figure 3. TUNEL staining of FLS. TUNEL-positive cells were shown in KOA synovia (black arrows) (magnification, x400). KOA, knee osteoarthritis; TUNEL, terminal deoxynucleotidyl transferase dUTP nick end labeling; FLS, fibroblast-like synoviocytes.

were firstly identified by $\mathrm{H} \& \mathrm{E}$ and immunostaining (Fig. 4). After the intervention of LPS and ATP, cell apoptosis was detected by flow cytometry (Fig. 5). The cell apoptosis rate in the LPS+ATP group was significantly higher than the normal group $(\mathrm{P}<0.01)$. Meanwhile, the NLRP1 and NLRP3 inflammasome-related proteins were upregulated (Figs. 6 and 7),

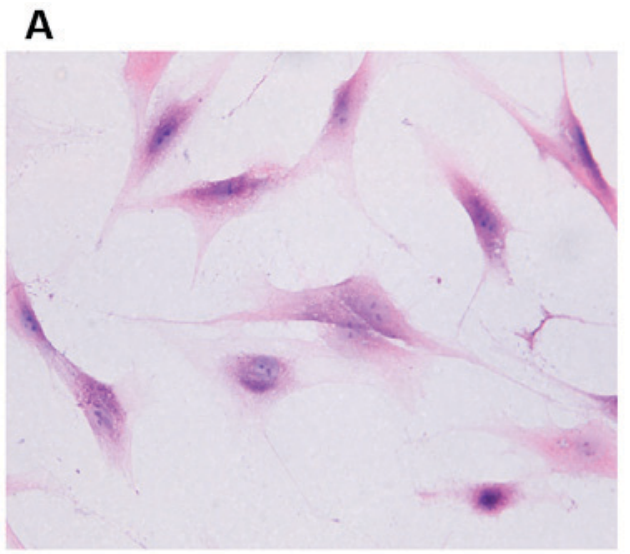

B

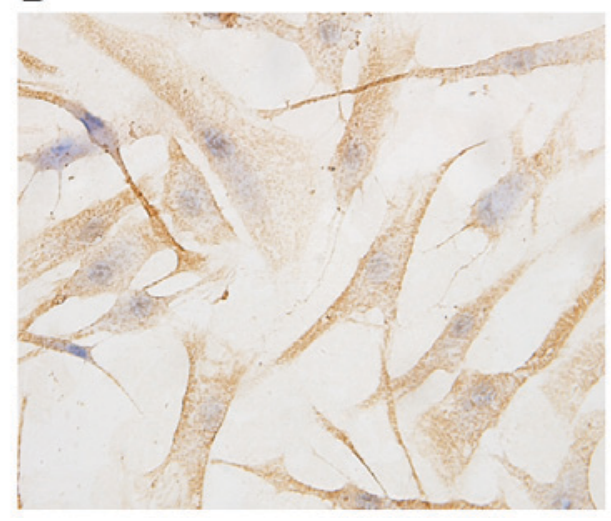

Figure 4. Histology analysis of FLS. (A) H\&E staining and (B) immunostaining (both magnification, x400). FLS, fibroblast-like synoviocyte.

which means the pyroptosis may take place during the subsequent LPS+ATP-induced inflammatory reactions.

NLRPI siRNA attenuates the LPS+ATP induced inflammasomes activation in FLS. The silencing effect of the NLRP1 siRNA was also confirmed by western blotting (Fig. 6A and B). The normal FLS were used as a normal control. The LPS-induced cell inflammation and pyroptosis were inhibited by the NLRP1 siRNA, and the expression of ASC, caspase-1, IL-18 and GSDMD in LPS+ATP and vehicle scrambled siRNA groups were significantly higher than the NLRP1 siRNA group (Fig. 6C and D), meanwhile the relative mRNA expression of IL-18 in NLRP1 siRNA group showed no significant alternation comparing with LPS+ATP and vehicle scrambled siRNA groups (Fig. 6E). The relative mRNA expression of ASC, caspase-1, IL-18 and GSDMD in LPS+ATP and vehicle scrambled siRNA groups were significantly higher than the NLRP1 siRNA group (Fig. 6E).

NLRP3 siRNA attenuates the LPS+ATP induced inflammasomes activation in FLS. The silencing effect of the NLRP3 siRNA were confirmed by western blotting (Fig. 7A and B). The normal FLS were used as a normal control. The LPS-induced cell inflammation and pyroptosis were inhibited by the NLRP3 siRNA, the protein and relative mRNA expression of ASC, caspase-1 and GSDMD expression in LPS+ATP and vehicle scramble siRNA group were significantly higher 
A

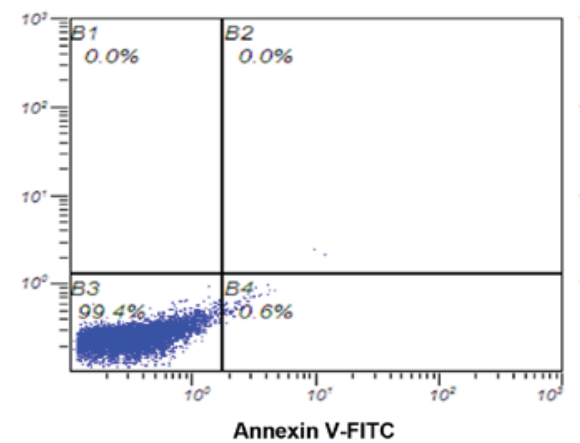

C

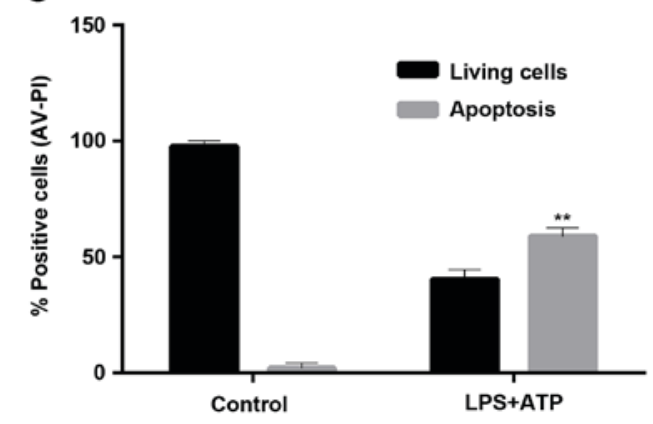

B

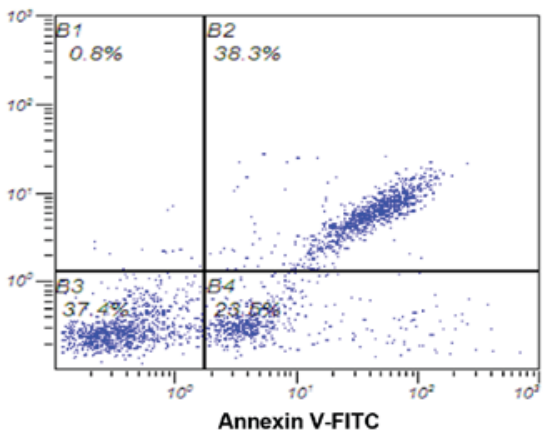

Annexin V-FITC

Figure 5. Flow cytometry analysis after the treatment of LPS+ATP. (A) cell apoptosis in control group; (B) cell apoptosis in LPS+ATP treatment group. (C) quantifications of apoptotic cells, ${ }^{* *} \mathrm{P}<0.01$ vs. control group. LPS, lipopolysaccharide.

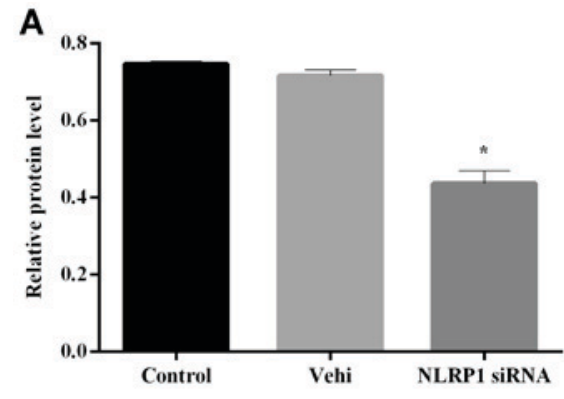

\section{C}

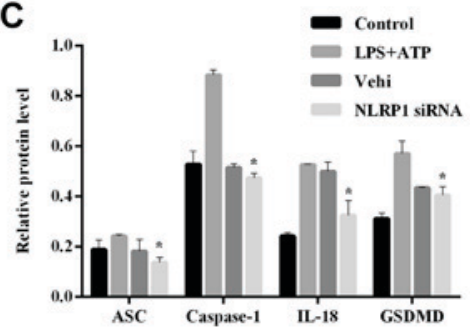

E
B

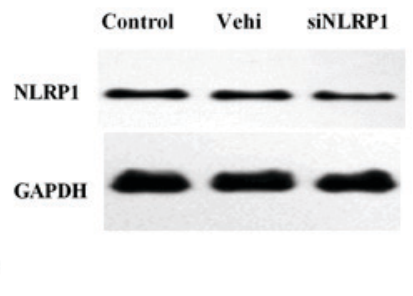

D

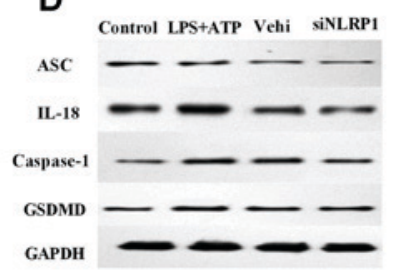

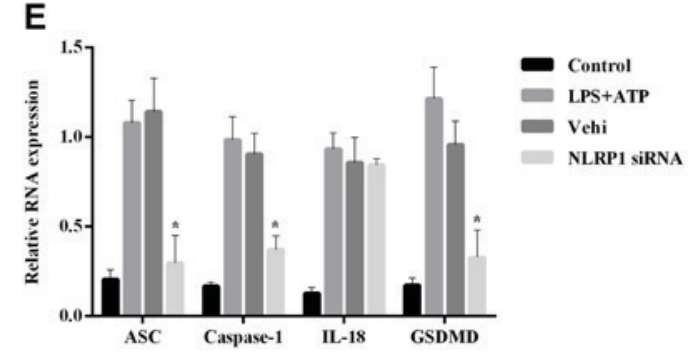

Figure 6. mRNA and protein expression of NLRP1 inflammasome components and downstream cytokines after NLRP1 siRNA transfection. FLSs were incubated with LPS+ATP for $7 \mathrm{~h}$. After that, NLRP1 siRNA was transfected. The silencing effect of the NLRP1 siRNA was also confirmed by western blotting (A, B). The mRNA and protein expression of NLRP1 inflammasome components (ASC, caspase-1) and downstream cytokines (GSDMD) were downregulated after the transfection of NLRP1 siRNA compared with the LPS+ATP group (C, D and E). The mRNA level of IL-18 between LPS+ATP group and NLRP1 siRNA group showed no significant alternation. "P<0.05 vs. LPS+ATP group. FLS, fibroblast-like synoviocyte; LPS, lipopolysaccharide; siRNA, small interfering RNA; ASC, apoptosis-associated Speck-like protein with a caspase-recruitment domain. 

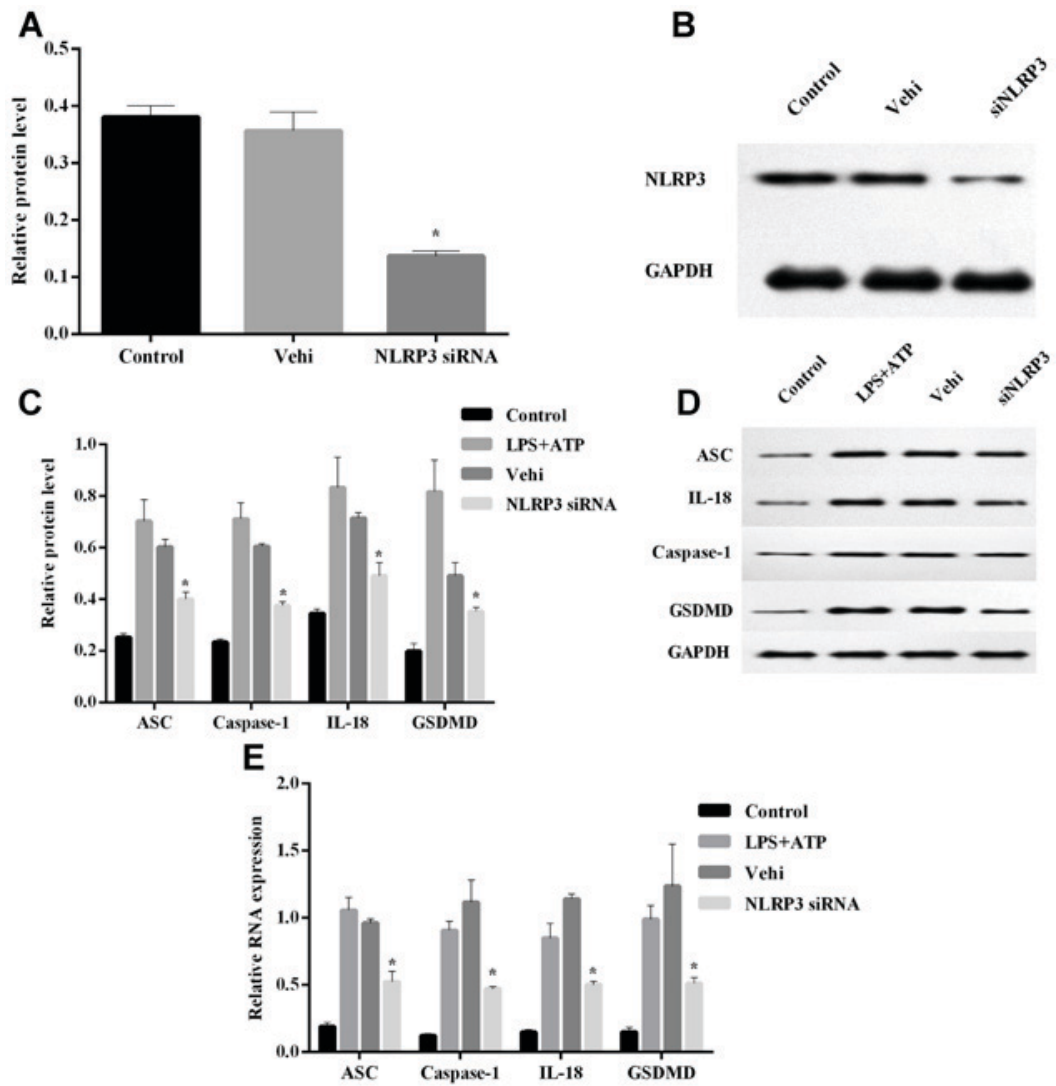

Figure 7. mRNA and protein expression of NLRP3 inflammasome components and downstream cytokines after NLRP3 siRNA transfection. FLSs were incubated with LPS+ATP for $7 \mathrm{~h}$. NLRP3 siRNA was transfected. The silencing effect of the NLR3 siRNA were also confirmed by western blotting (A and B). The mRNA and protein expression of NLRP3 inflammasome components (ASC, caspase-1) and downstream cytokines (IL-18, GSDMD) were downregulated after the treatment of NLRP3 siRNA compared with the LPS+ATP group (C, D and E). ${ }^{*} \mathrm{P}<0.05$ vs. LPS+ATP group.

than the NLRP3 siRNA group, which may reveal the fact that NLRPs are crucial determinant in LPS-induced FLS pyroptosis (Fig. 7C-E).

\section{Discussion}

In the present study, the expression of NLRP1 and NLRP3 inflammasomes were confirmed to be up-related in the tissue of KOA patients. Meanwhile, in vitro experiments demonstrated that after the treatment of LPS+ATP, NLRP1 and NLRP3 siRNAs may lead to a significantly reduction of inflammasome-related mRNA and protein expression compared with those with normal FLS. These findings indicated that both NLRP1 and NLRP3 act as important inducers of FLS pyroptosis.

Although the etiology and pathogenesis of KOA has not yet been fully defined, the cytokines IL- $1 \beta$ and IL-18 have received extensive attention to the central pathogenesis of KOA. In a previous study, Denoble et al (22) found that uric acid can activate NLRP3 inflammasome, which results in the increase of IL-18 and IL-1 $\beta$. The uric acid and IL-18 and IL-1 $\beta$ in synovial fluid were closely correlated with the severity of KOA, suggesting that inflammasome signaling pathways may be involved in the development and progression of KOA.

Activation of NLR inflammasomes and induction of pyroptosis may require a two-step mechanism. The first step is the priming step. Pro-inflammatory mediators, such as pro-IL-1 $\beta$, NLRP1/3 and caspase family members, are transcriptionally generated. The second step is the activation step, where the inflammasomes are assembled and caspase-1 is activated. Active caspase- 1 proteolytically matures pro-IL- $1 \beta$ and proIL-18 and therefore induces pyroptosis partially through cleavage of gasdermin D (GSDMD). Unlike caspase-1, caspase-11 is activated through direct sensing of intracellular LPS in the cytoplasm of infected cells, and promotes pyroptosis through GSDMD cleavage. Pyroptosis features rapid plasma membrane rupture, resulting in the release of intracellular pathogens and pro-inflammatory mediators, including IL-1 $\beta$, IL-18 and other inflammatory cytokines.

NAcht leucine-rich-repeat protein 1 (NLRP1, also known as NALP1), a regulator of the innate immune response expressed in various cell types. The NLRP1/IL-1 $\beta$ axis has been reported to be associated with autoimmunity (23). The assembly of the NLRP1 inflammasome and the subsequent activation of caspase-1 cleaves the inactive IL- $1 \beta$ precursor to the bioactive IL-1 $\beta$, thus stimulating downstream inflammatory responses (24). Some clinical evidences also suggest that NLRP1 polymorphisms are also involved in the predisposition to rheumatoid arthritis and other autoimmune diseases (25).

As mentioned above, the NLRP3 inflammasome is receiving increasing attention in the recent researches since it participates various diseases (26). Multiple mechanisms have been proposed to activate NLRP3, including bacterial, viral and fungal agents, $\mathrm{K}+$ efflux, lysosomal destabilization, pore formation in the plasma membrane, mitochondrial damage, the production of reactive oxygen species, $\mathrm{Ca}^{2+}$ influx, and 
cell swelling. In the progression of KOA, chronic inflammation should be the predominant pathological change in the synovial tissues. Various exogenous and endogenous stimuli are recognized by the NLRP3 receptor and trigger the inflammasome activation (8). In the recent study, Shi et al (27) revealed that increased LPS and ATP in joint-space may promote KOA by NLRP3 Inflammasome, they showed that inhibiting NLRP3 inflammasome may exert protective effect. Our result supported the same fact that NLRP3 inflammasome is a crucial part of KOA, especially in the pyroptosis of FLS.

In the present study, IL-18 mRNA expression showed no significant alternation after the treatment of NLRP1 siRNA. The possible reason should be that IL-18 is not mediated by NLRP1 inflammasome, the IL-18 protein expression is regulated by some other inflammatory signal pathways. Besides, more in vivo experiments should be undertaken in the future, in order to approach more molecular mechanisms in the treatment of KOA inflammation.

Inflammasomes are intracellular pattern recognition receptors (PRRs) that play an important regulatory role in stimulating and regulating immune and inflammatory responses. Constantly updated data show that inflammasome/caspase-1/IL-1 $\beta$ inflammatory pathways involved in the occurrence and development of a variety of inflammatory diseases. Based on the data obtained from patients and in vitro cells, we concluded that both NLRP1 and NLRP3 inflammasomes are highly involved in the FLS inflammation and pyroptosis. Moreover, inhibition of NLRP1 and NLRP3 led to a remarkable reduction of pyroptosis-related cytokines. But currently, further study is required to determine the effect of NLRP1 and NLRP3 inflammasome inhibitors/blockers in vivo. Our further clinical study will focus on the application of inflammasome/caspase-1/IL-1 $\beta$ pathway inhibitors in the treatment of KOA. Its mechanism will provide new ideas and means for understanding and treating KOA-related inflammatory diseases.

\section{Acknowledgements}

The present study was supported by the National Natural Science Foundation of China (81573993).

\section{References}

1. Higgs R: Osteoarthritis: Concentrated efforts to detect early OA. Nat Rev Rheumatol 6: 616, 2010.

2. Okubo M and Okada Y: Destruction of the articular cartilage in osteoarthritis. Clin Calcium 23: 1705-1713, 2013 (In Japanese)

3. Wright AA, Cook C and Abbott JH: Variables associated with the progression of hip osteoarthritis: A systematic review. Arthritis Rheum 61: 925-936, 2009.

4. Kochukov MY, McNearney TA, Yin H, Zhang L, Ma F, Ponomareva L, Abshire S and Westlund KN: Tumor necrosis factor-alpha (TNF-alpha) enhances functional thermal and chemical responses of TRP cation channels in human synoviocytes. Mol Pain 5: 49, 2009.

5. Fernandes ES, Russell FA, Spina D, McDougall JJ, Graepel R, Gentry C, Staniland AA, Mountford DM, Keeble JE, Malcangio M, et al: A distinct role for transient receptor potential ankyrin 1, in addition to transient receptor potential vanilloid 1, in tumor necrosis factor $\alpha$-induced inflammatory hyperalgesia and Freund's complete adjuvant-induced monarthritis. Arthritis Rheum 63: 819-829, 2011.

6. El Karim I, McCrudden MT, Linden GJ, Abdullah H, Curtis TM, McGahon M, About I, Irwin C and Lundy FT: TNF- $\alpha$-induced p38MAPK activation regulates TRPA1 and TRPV4 activity in odontoblast-like cells. Am J Pathol 185: 2994-3002, 2015.
7. Jotanovic Z, Mihelic R, Sestan B and Dembic Z: Role of interleukin-1 inhibitors in osteoarthritis: An evidence-based review. Drugs Aging 29: 343-358, 2012.

8. Loreto C, Musumeci G and Leonardi R: Chondrocyte-like apoptosis in temporomandibular joint disc internal derangement as a repair-limiting mechanism. An in vivo study. Histol Histopathol 24: 293-298, 2009.

9. Musumeci G, Castrogiovanni P, Loreto C, Castorina S, Pichler K and Weinberg AM: Post-traumatic caspase-3 expression in the adjacent areas of growth plate injury site: A morphological study. Int J Mol Sci 14: 15767-15784, 2013.

10. Puzzo D, Loreto C, Giunta S, Musumeci G, Frsaca G, Podda MV, Arancio O and Palmeri A: Effect of phosphodiesterase-5 inhibition on apoptosis and beta amyloid load in aged mice. Neurobiol Aging 35: 520-531, 2014.

11. Musumeci G, Cstrogiovanni P, Travato FM, Weinberg AM, Al-Wasiyah MK, Algahtani MH and Mobasheri A: Biomarkers of chondrocyte apoptosis and autophagy in osteoarthritis. Int J Mol Sci 16: 20560-20575, 2015.

12. Tschopp J and Schroder K: NLRP3 inflammasome activation: The convergence of multiple signaling pathways on ROS production? Nat Rev Immunol 10: 210-215, 2010.

13. Martinon F, Burns K and Tschopp J: The inflammasome: A molecular platform triggering activation of inflammatory caspases and processing of proIL-beta. Mol Cell 10: 417-426, 2002.

14. Galluzzi L, Vitale I, Abrams JM, Alnemri ES, Baehrecke EH, Blagosklonny MV, Dawson TM, Dawson VL, El-Deiry WS, Fulda S, et al: Molecular definitions of cell death subroutines: Recommendations of the Nomenclature Committee on Cell Death 2012. Cell Death Differ 19: 107-120, 2012.

15. Zhuang Y, Ding G, Zhao M, Bai M, Yang L, Ni J, Wang R, Jia Z, Huang S and Zhang A: NLRP3 inflammasome mediates albumin-induced renal tubular injury through impaired mitochondrial function. J Biol Chem 289: 25101-25111, 2014

16. Fann DY, Lim YA, Cheng YL, Lok KZ, Chunduri P, Baik SH, Drummond GR, Dheen ST, Sobey CG, Jo DG, et al: Evidence that NF- $\kappa \mathrm{B}$ and MAPK signaling promotes NLRP inflammasome activation in neurons following ischemic stroke. Mol Neurobiol: Jan 14, 2017 (Epub ahead of print).

17. Li-Jessen NYK, Powell M, Choi AJ, Lee BJ and Thibeault SL: Cellular source and proinflammatory roles of high-mobility group box 1 in surgically injured rat vocal folds. Laryngoscope 127: E193-E200, 2017.

18. Wang X, Guo Y, Wang C, Yu H, Yu X and YuH:MicroRNA-142-3p inhibits chondrocyte apoptosis and inflammation in osteoarthritis by targeting HMGB1. Inflammation 39: 1718-1728, 2016.

19. Musumeci G, Castrogiovanni P, Mazzone V, Szychlinska MA, Castorina S and Loreto C: Histochemistry as a unique approach for investigating normal and osteoarthritic cartilage. Eur J Histochem 58: 2371, 2014.

20. Tong S, Liu J and Zhang C: Platelet-rich plasma inhibits inflammatory factors and represses rheumatoid fibroblast-like synoviocytes in rheumatoid arthritis. Clin Exp Med 17: 441-449, 2017.

21. Zhang W, Tao A, Lan T, Cepinskas G, Kao R, Martin CM and Rui T: Carbon monoxide releasing molecule-3 improves myocardial function in mice with sepsis by inhibiting NLRP3 inflammasome activation in cardiac fibroblasts. Basic Res Cardiol 112: 16, 2017.

22. Denoble AE, Huffman KM, Stabler TV, Kelly SJ, Hershfield MS, McDeniel GE, Coleman RE and Kraus VB: Uric acid is a danger signal of increasing risk for osteoarthritis through inflammasome activation. Proc Natl Acad Sci USA 108: 2088-2093, 2011.

23. Levandowski CB, Mailloux CM, Ferrara TM, Gowan K, Ben S, Jin Y, McFann KK, Holland PJ, Fain PR, Dinarello CA and Spritz RA: NLRP1 haplotypes associated with vitiligo and auto-immunity increase IL-1 $\beta$ processing via the NLRP1 inflammasome. Proc Natl Acad Sci USA 110: 2952-2956, 2013.

24. van de Veerdonk FL, Netea MG, Dinarello CA and Joosten LA: Inflammasome activation and IL-1 $\beta$ and IL-18 processing during infection. Trends Immunol 32: 110-116, 2011.

25. Sui J, Li H, Fang Y, Liu Y, Li M, Zhong B, Yang F, Zou Q and Wu Y: NLRP1 gene polymorphism influences gene transcription and is a risk factor for rheumatoid arthritis in han chinese. Arthritis Rheum 64: 647-654, 2012.

26. Strowig T, Henao-Mejia J,Elinav E and Flavell R: Inflammasomes in health and disease. Nature 481: 278-286, 2012.

27. Shi J, Zhao W, Ying H, Zhang Y, Du J, Chen S, Li J and Shen B: Estradiol inhibits NLRP3 inflammasome in fibroblast-like synoviocytes activated by lipopolysaccharide and adenosine triphosphate. Int J Rheum Dis: Nov 3, 2017 (Epub ahead of print). 